\title{
BREAKING THE MOULD
}

When you think about it, many materials are alive - not just, say, those of tissue engineering, but materials intended for 'inorganic' applications. As librarians know too well, paper is an ideal host for moulds. There's plenty of biological activity going on, I fear, in my floorboards. Buildings and roads are coated and threaded with plant matter. Even metals can support, and be corroded by, bacteria. Structurally and functionally, such materials must be considered organic-inorganic composites.

Why not turn that problem to advantage? Indeed, we already do. For example, some buildings support turf roofs as an integral part of their ecology. Gerber et al. have now described what might be considered the microbiological analogue of the turf roof: coating materials that host colonies of fungi in an active 'living surface' (L. C. Gerber, F. M. Koehler, R. N. Grass \& W. J. Stark Proc. Natl Acad. Sci. USA 109, 90-94; 2012).

This concept sets aside the notion of biomimesis in favour of a direct utilization of the responsive, adaptive capabilities of organisms themselves, in much the same way as industrial catalysis might be performed by vats of engineered bacteria. Gerber et al. point out that Camembert and
Roquefort cheeses are in a sense living materials, hosting Penicillium moulds that supply their distinctive aroma and flavour.

As biotechnologists are well aware, the challenge of using living organisms in engineering contexts is keeping them alive. Fungi are among the most resourceful of organisms in that respect, because many already have highly developed ways of adapting to changing environments, such as the formation of spores to hibernate when moisture or nutrients are scarce. Some are also able to migrate when exposed to stress.

Gerber et al. have used Penicillium roquefort $i$ itself as the active agent of their living materials. This mould feeds aerobically on sugars. The researchers culture it in a layer of agar gel around $300 \mu \mathrm{m}$ thick when dry. They cover this with a nanoporous polycarbonate membrane $10 \mu \mathrm{m}$ thick. The average pore diameter of $\sim 400 \mathrm{~nm}$ is sufficient to allow easy permeation of gas, nutrients and secondary metabolites, while small enough to prevent the fungi from escaping.

When 'food' (glucose) was spilled on the surface, the fungi grew and consumed it within several days. Once the sugar level fell below the threshold of detectability, the fungi sporulated and entered hibernation, but could be

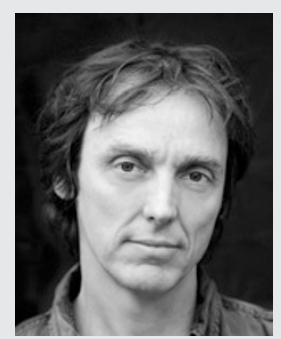

PHILIP BALL

reactivated by adding more sugar days later. In this dormant state the microorganisms can be expected to survive for at least several weeks. Cleaning the surface with ethanol or soap did not seem to harm the fungi; only prolonged dehydration did permanent damage.

The composite, which Gerber et al. call 'the first eating material', can be made in large areas - the researchers have made sheets of up to $500 \mathrm{~cm}^{2}$. They suggest that penicillin-producing moulds might be used to make living antibacterial surfaces that are selfsterilizing on demand. The materials could also be used in packaging, for example to protect food while cleaning up its own leaks. In the face of complex, changing environmental stimuli, these living films might even display the capacity for evolutionary adaptation. 